\title{
Design and control of a pneumatic musculoskeletal biped robot
}

\author{
Xizhe Zang*, Yixiang Liu, Xinyu Liu and Jie Zhao \\ State Key Laboratory of Robotics and System, Harbin Institute of Technology, Harbin, Heilongjiang, \\ China
}

\begin{abstract}
.
BACKGROUND: Pneumatic artificial muscles are quite promising actuators for humanoid robots owing to their similar characteristics with human muscles. Moreover, biologically inspired musculoskeletal systems are particularly important for humanoid robots to perform versatile dynamic tasks.

OBJECTIVE: This study aims to develop a pneumatic musculoskeletal biped robot, and its controller, to realize human-like walking.

METHODS: According to the simplified musculoskeletal structure of human lower limbs, each leg of the biped robot is driven by nine muscles, including three pairs of monoarticular muscles which are arranged in the flexor-extensor form, as well as three biarticular muscles which span two joints. To lower cost, high-speed on/off solenoid valves rather than proportional valves are used to control the muscles. The joint trajectory tracking controller based on PID control method is designed to achieve the desired motion. Considering the complex characteristics of pneumatic artificial muscles, the control model is obtained through parameter identification experiments.

RESULTS: Preliminary experimental results demonstrate that the biped robot is able to walk with this control strategy.

CONCLUSION: The proposed musculoskeletal structure and control strategy are effective for the biped robot to achieve human-like walking.
\end{abstract}

Keywords: Biped robot, musculoskeletal structure, pneumatic artificial muscles, PID control

\section{Introduction}

Humanoid robots have been a fascinating hotspot of research in the area of robotics for many years because of their resemblance to humans. There are two crucial aspects in the designing and developing of humanoid robots. The first one is selecting appropriate actuators. Recent requirements for actuators, mainly for their performance, performance to weight ratio, as well as compactness with minimal internal structure and with the integration of main functional parts, lead to intensive application of new and nontraditional solutions. One of the possible approaches to novel actuators is Pneumatic Artificial Muscles, or PAMs [1], which generate traction force when they contract, just like human muscles [2]. PAMs are becoming more and more widely used in humanoid robots due to their variable-stiffness spring-like characteristics, physical flexibility, and very light weight compared to their electrical counterparts [3]. The second aspect is anthropomorphic musculoskeletal structure, including human-like body structure, arrangements of redundant muscles, and adjustable joint softness [4]. The human musculoskeletal system

\footnotetext{
${ }^{*}$ Corresponding author: Xizhe Zang, State Key Laboratory of Robotics and System, Harbin Institute of Technology, Harbin, Heilongjiang, China. Tel.: +86 045186413382; Fax: +86 045186414538; E-mail: zangxizhe@ hit.edu.cn.
} 
gives human the ability to move in a huge variety of environments. Thus, biologically inspired designs are particularly important for humanoid robots to perform versatile dynamic tasks. By developing and using such an actual musculoskeletal humanoid robot as a detail human body simulator in the real world, researchers can understand human motor function and human motor control better [5].

Up to present, a lot of humanoid robots driven by PAMs have been proposed. Shadow Robot Company developed a wooden leg-skeleton to study the movements of human lower limbs. But it cannot walk. It is only able to stand up controlled by a simple fuzzy rule-based controller. Dirk et al. built a planar walking biped robot Lucy [6]. Each joint of Lucy, driven by two PAMs in antagonistic setup, is actively controlled, enabling it to walk at different speeds and step-lengths on a treadmill [7,8]. However, this robot only uses monoarticular muscles, making it different with human lower limbs which incorporate both monoarticular and biarticular muscles. Hosoda et al. added biarticular muscles to their developed pneumatic actuated biped robots [9-15]. These robots can be controlled to walk by a simple open-loop controller, which gives a certain activation pattern to each muscle within one walking cycle. But the passivity-based controller causes the loss of some controllability to the realized gait. Apart from bipedal walking robots, several hopping robots and jumping robots with artificial musculoskeletal systems have also been designed by researchers [16-20].

In this paper, we aim to develop a pneumatic biped robot which synthesizes the virtue of the above researches - the musculoskeletal structure and controllability instantaneously to realize human-like walking. On one hand, to mimic the musculoskeletal structure of humans, both monoarticular and biarticular muscles are arranged in the biped robot, making it a compliant and redundant system. On the other hand, PAMs have very complex characteristics such as high nonlinearity, hysteresis and time delay, and their current theoretical models are too complicated [21-23]. To simplify the control of PAMs, we obtain the control model through identification experiments without formally dealing with their complicated dynamics. Based on the control model, the robot walking controller is designed and then tested through walking experiments.

This paper is organized as follows. Firstly, the design of the pneumatic musculoskeletal biped robot is introduced. Secondly, the control scheme for the biped robot is presented. Finally, preliminary walking experiment results of the robot are shown.

\section{Design of the biped robot}

\subsection{The biped robot}

A musculoskeletal biped robot actuated by PAMs is designed in this study. Figure 1 shows the appearance of the robot. The robot is composed of two identical legs, each having a thigh, a shank and a flat foot. In order to avoid unnecessary complexity regarding design and control, the movement of the robot is restricted to the sagittal plane. Therefore, all parts are connected by one-dimensional pin joints, creating the hip, knee and ankle joint. In total, the robot has six internal degrees of freedom (DOF). To make the robot anthropopathic, the length of each link is expected to be of similar ratio as that of humans. The robot is $1100 \mathrm{~mm}$ high and $350 \mathrm{~mm}$ wide. The lengths of thigh and shank are 440 and $430 \mathrm{~mm}$ respectively. The structure of the robot is made of POM, a kind of polymer with high mechanical strength and low density.

The McKibben type PAMs produced by FESTO Corporation are adopted for joint driving. The maximum contraction ratio of the muscles is approximately $25 \%$, while the maximum working pressure is 


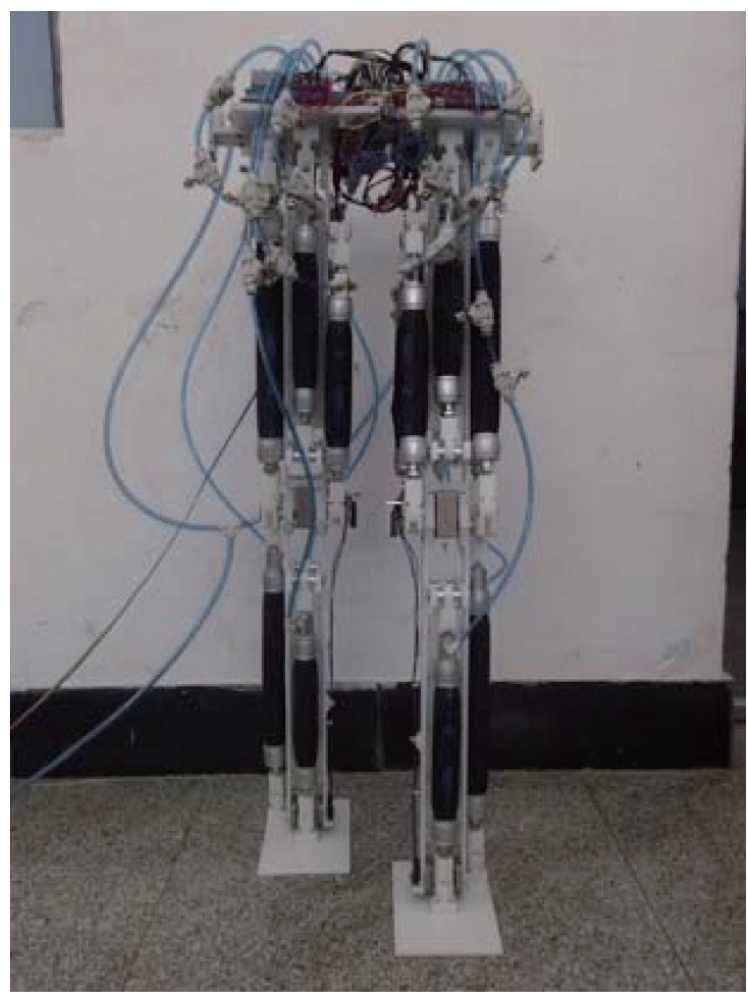

Fig. 1. The biped robot.

0.6 MPa. The muscle radius is $20 \mathrm{~mm}$. The force provided by the muscle is dependent on the internal pressure. Because of the PAMs, the robot has a lightweight and flexible body.

The biped robot system is composed of lithium-ion battery, micro controller, pneumatic solenoid valves, and angle sensors within its body. Compressed air of which the maximum pressure is $1.2 \mathrm{MPa}$ is supplied to the valves from an external compressor via an air regulator. The robot is self-contained except this compressor. 18 PAMs are equipped on the robot totally, making it quite expensive to use proportional servo valves to control the muscles. What's more, there is no enough space to install these relatively big-sized valves. In order to realize a compact, rapid and accurate control, high-speed on/off solenoid valves VFS1320 with small size and light weight produced by SMC Corporation are selected. The maximum operation frequency of the valves is $600 \mathrm{~Hz}$ with a short response time of less than $20 \mathrm{~ms}$. And the working pressure ranges from 0.15 to $1 \mathrm{MPa}$, which is sufficient for the use of PAMs. The valves have three positions: supplying air to the valve, expelling air to the atmosphere, and keeping closed. Angle sensors with a resolution of 12 bits made by Novotechnik Corporation are adopted to measure joint angles. The controller to drive the solenoid valves is a 16-bit single-chip microcomputer H8/3069 of Renesas Technology Corporation. The micro controller sends operation commands, open or close, to the valves, and receives data from the angle sensors.

\subsection{Musculoskeletal system}

As a significant characteristic, the biped robot is designed based on the biological structure of human lower limbs. For humans, joint movements are completed based on the complementary functions 


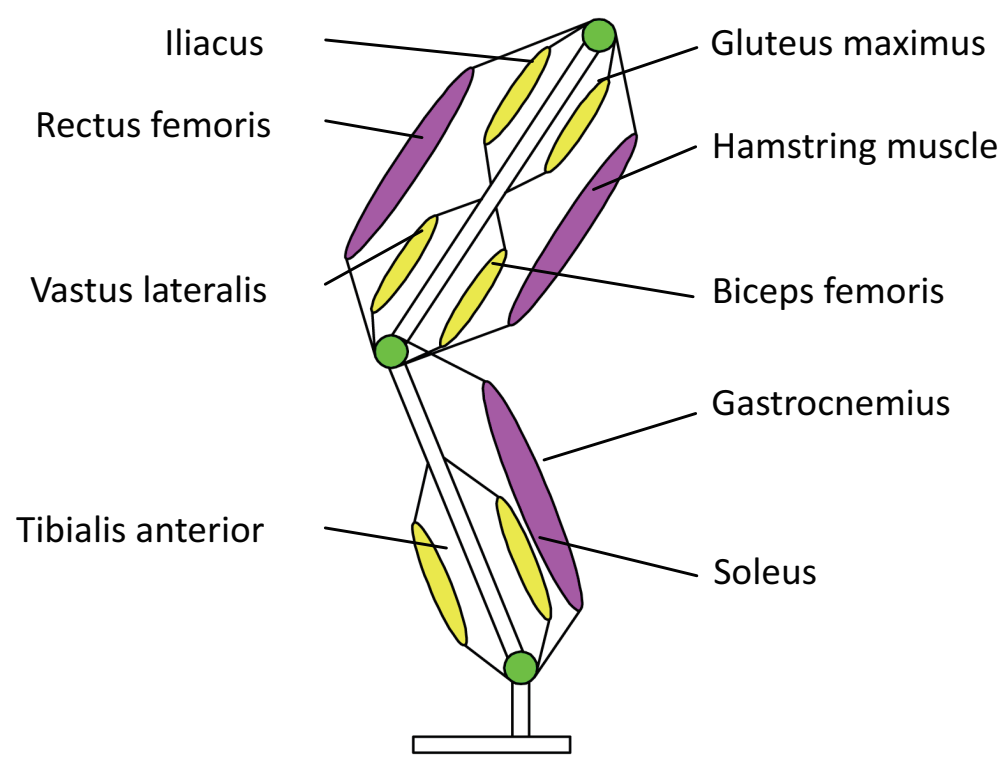

Fig. 2. Musculoskeletal model of the biped robot.

of monoarticular and biarticular muscles. Monoarticular muscles can only pull and mainly act as unidirectional force generators. To realize bidirectional motion, two monoarticular muscles are arranged in the flexor-extensor form. Biarticular muscles that span two joints play a crucial role in human walking. They are capable of transferring energy between joints by coupling joint motions [24,25]. Figure 2 shows the simplified musculoskeletal model of the biped robot. Each leg with three rotating joints contains nine muscles including six monoarticular muscles and three biarticular muscles, each of which represents groups of synergist muscles across the joints. Consequently, the biped robot is a flexible and coordinative redundant system.

Monoarticular muscles on the hip joint include gluteus maximus (GM), which extends the hip, and iliacus (IL), which flexes the hip. Vastus lateralis (VL) extends the knee joint, and biceps femoris (BF) flexes the knee joint. The ankle joint is extended by soleus (SO) and flexed by tibialis anterior (TA). Biarticular muscles include rectus femoris (RF) and hamstring muscle (HM), which both span the hip and knee joint, as well as gastrocnemius (GA), which spans the knee and ankle joint.

\section{Design of the control system}

\subsection{Control scheme}

The main idea of the proposed control system is joint trajectory tracking. For a joint driven by antagonistically arranged muscles, joint movements are achieved through contraction of the agonistic muscle and relaxation of the antagonistic muscle. Consequently, to achieve the desired joint angle, we only need to control the muscles to contract and stretch to the desired lengths. And to achieve the desired muscle lengths, we only need to control the duration of supplying air into the muscle and expelling air to the atmosphere. Thus, in this way, the control of joint angle is eventually translated into the control of action time of solenoid valves. 


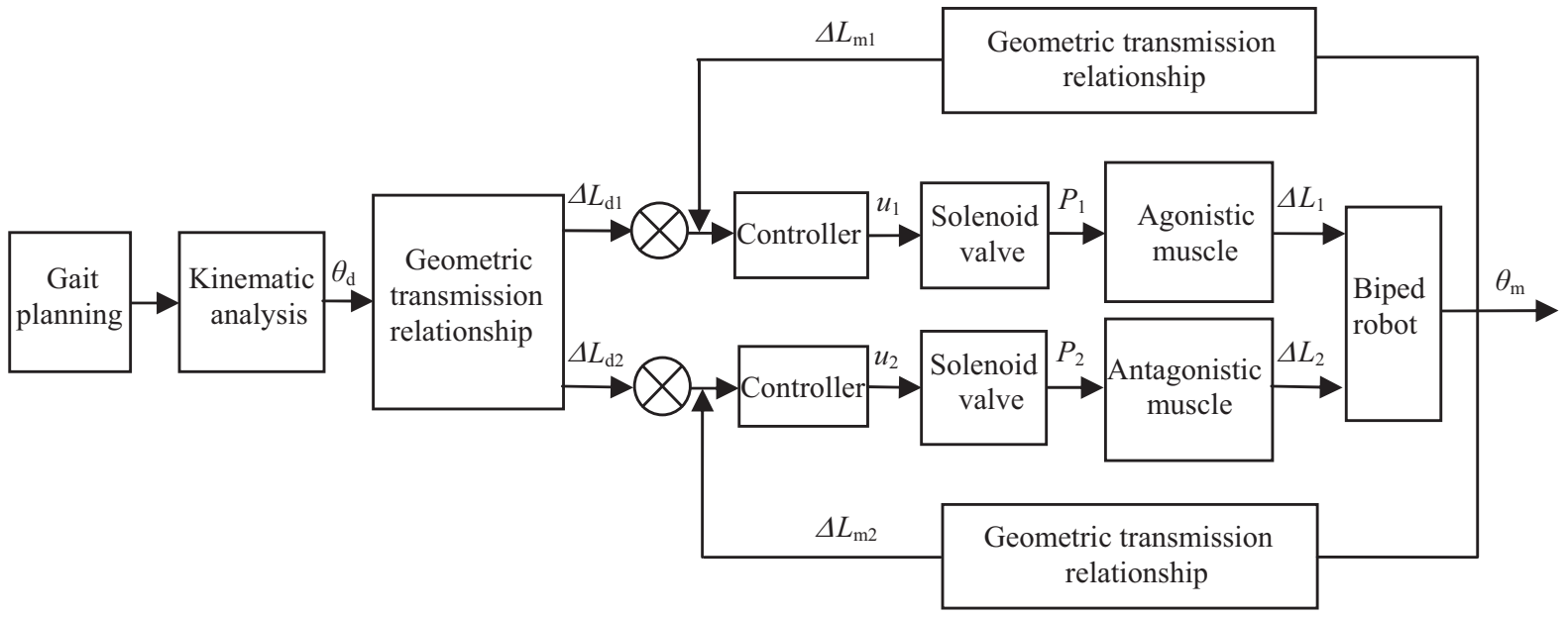

Fig. 3. Control scheme of the biped robot.

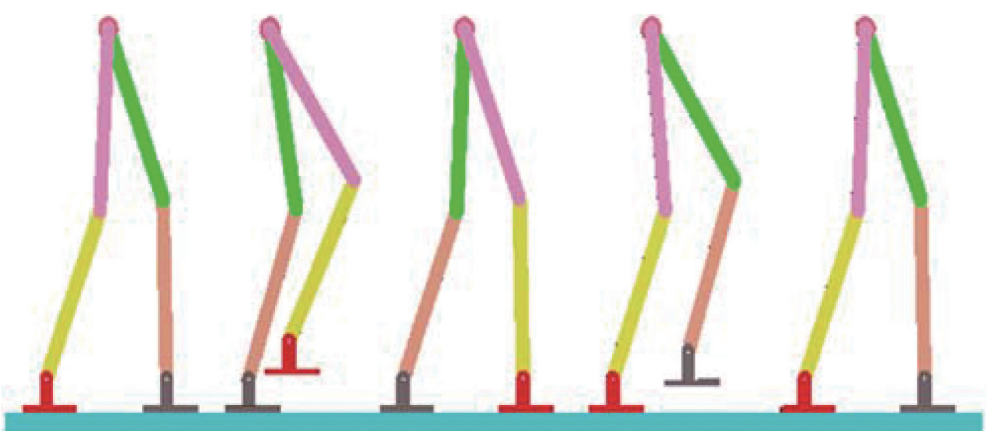

Fig. 4. Walking gait with flat feet.

Figure 3 presents the control block diagram of the biped robot. First of all, given the walking parameters including step length, step height, cycle time etc., the desired gait is planned based on ZMP criterion. Then, the joint angles are calculated using inverse kinematic analysis. After that, the joint angles are transformed into corresponding muscle lengths according to the geometric transmission relationship of the joints. At the end, general PID control method is adopted to control each PAM to reach the desired length. It will be difficult to directly measure the muscle length using linear displacement sensor. However, the muscle length can be derived from the geometric transmission relationship based on the measured joint angle. Therefore, the joint angles are used as the feedback signal of the PID controller. And the control model of the PAM is obtained through identification experiments.

\subsection{Gait planning}

The walking gait of biped robots with flat feet is usually composed of two fully actuated phases: the single support phase and the double support phase. During the single support phase, the support leg supports the whole robot while the swing leg moves forward. When the swing foot touches the ground, the double support phase starts. During this phase, the support point is transferred to the swing leg which becomes the support one. Figure 4 shows the walking gait of biped robots with flat feet. 


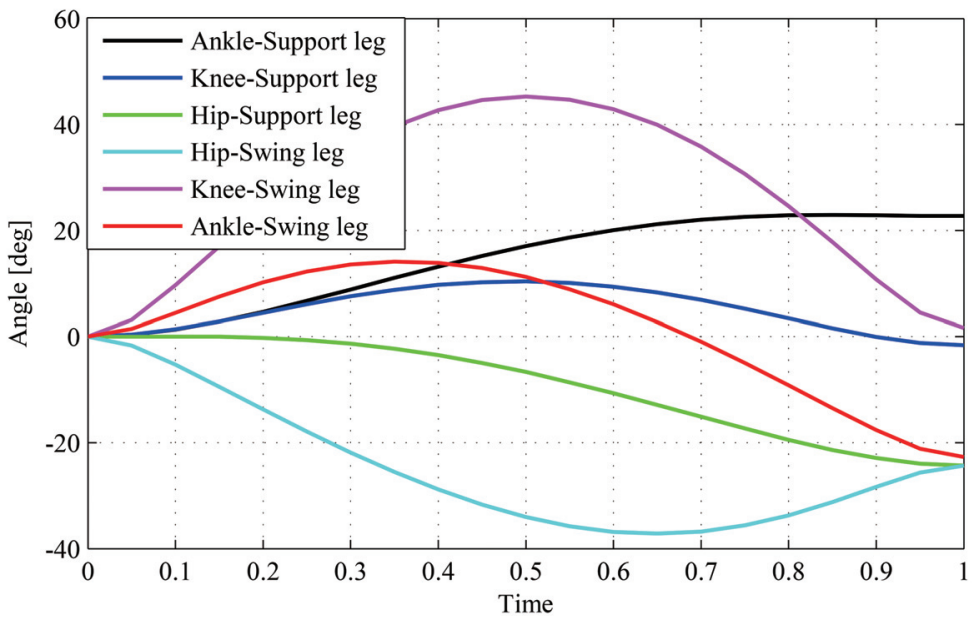

Fig. 5. Joint trajectories.

In this study, point-to-point path planning method is adopted to realize the desired walking gait on the base of ZMP criterion. According to the given walking parameters, the positions of several key intermediate points of the hip and feet are obtained. And the whole trajectories are derived using polynomials respecting the continuity of position, velocity and acceleration. Then joint angles of the robot are calculated using the inverse kinematic analysis, illustrated in Fig. 5.

\subsection{Geometric transmission relationship}

The geometric transmission relationship can be obtained according to the mechanical structure of the robot. Figure 6 presents the installation diagram of PAMs. In this figure, $\theta_{i}$ is the joint rotation angle, $r_{i}$ is the action radius of muscles, and $h_{i}$ is the distance between the joint and the articulation point of muscles. Besides, the initial mounting length of muscles and the difference between the instantaneous length and the initial mounting length are indicated as $L$ and $\Delta L$. Then the relationship between $\Delta L$ and $\theta_{i}$ can be expressed by the following equations.

$$
\begin{aligned}
& \Delta L_{T A}=\sqrt{\left(h_{2} \cos \theta_{1}-r_{1} \sin \theta_{1}-h_{1}\right)^{2}+\left(h_{2} \sin \theta_{1}+r_{1} \cos \theta_{1}-r_{1}\right)^{2}}-L_{T A} \\
& \Delta L_{S O}=\sqrt{\left(h_{2} \cos \theta_{1}+r_{1} \sin \theta_{1}-h_{1}\right)^{2}+\left(h_{2} \sin \theta_{1}-r_{1} \cos \theta_{1}+r_{1}\right)^{2}}-L_{S O} \\
& \Delta L_{V L}=\sqrt{\left(h_{4} \cos \theta_{2}+r_{2} \sin \theta_{2}-h_{3}\right)^{2}+\left(-h_{4} \sin \theta_{2}+r_{2} \cos \theta_{2}-r_{2}\right)^{2}}-L_{V L} \\
& \Delta L_{B F}=\sqrt{\left(h_{4} \cos \theta_{2}-r_{2} \sin \theta_{2}-h_{3}\right)^{2}+\left(-h_{4} \sin \theta_{2}-r_{2} \cos \theta_{2}+r_{2}\right)^{2}}-L_{B F} \\
& \Delta L_{I L}=\sqrt{\left(h_{2} \cos \theta_{1}-r_{1} \sin \theta_{1}+h_{1}\right)^{2}+\left(h_{2} \sin \theta_{1}+r_{1} \cos \theta_{1}-r_{1}\right)^{2}}-L_{I L} \\
& \Delta L_{G M}=\sqrt{\left(h_{2} \cos \theta_{1}+r_{1} \sin \theta_{1}+h_{1}\right)^{2}+\left(h_{2} \sin \theta_{1}-r_{1} \cos \theta_{1}+r_{1}\right)^{2}}-L_{G M}
\end{aligned}
$$




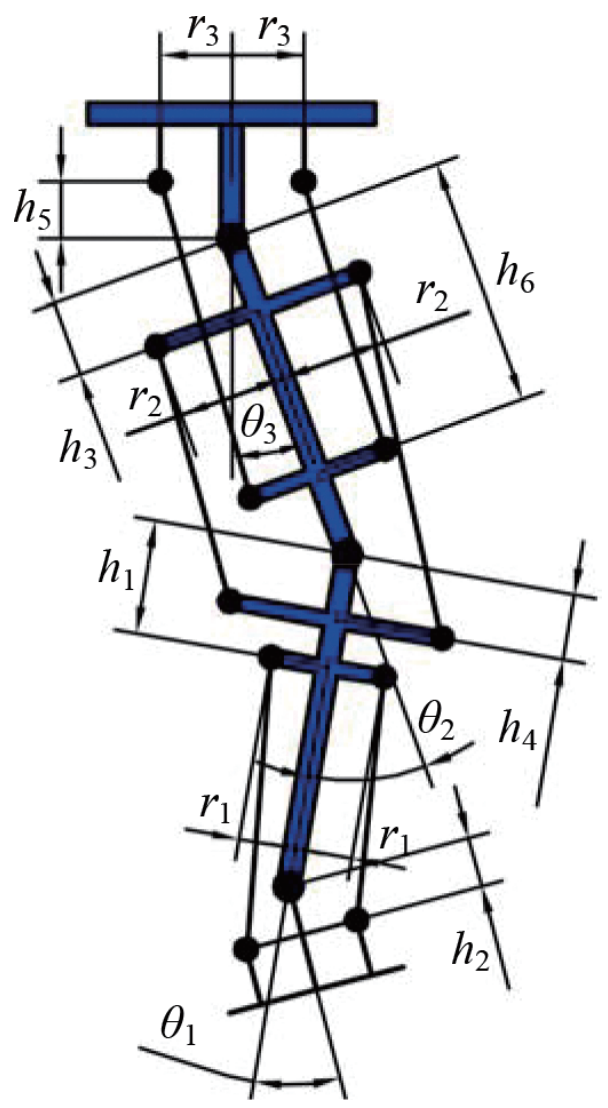

Fig. 6. Installation diagram of PAMs.

In these equations, the subscripts of $L$ and $\Delta L$ are the abbreviations for the scientific names of the monoarticular muscles mentioned earlier in this paper. The transmission relationship of biarticular muscles can be solved in the same way.

\subsection{Identification experiments}

The most important issue for designing the joint PID controller is to get the control models of the PAM including pressurizing and depressurizing process. The most popular mathematical models are very complicated. So we try to get a simplified empirical model through experiment.

In this experiment, we aim to derive the relationship between the action time of solenoid valve and muscle length under certain working pressure, for instance, 0.6 MPa. Initially, the PAM has the real length at zero gauge pressure. Then compressed air at the pressure of $0.6 \mathrm{MPa}$ is supplied to the muscle through a solenoid valve, causing the muscle to contract until reaching its limit position. After that, the air is exhausted outside of the muscle, making the muscle stretch to its original length. During this process, the contraction length of the muscle changing over time is measured, and then plotted in Matlab. The control models of pressurizing and depressurizing process are derived respectively based on the experimental results.

The black curve in Fig. 7 shows the measured contraction length of the muscle during the pressurizing process. It's not hard to find that the curve is very similar to the step response of a second-order system. 


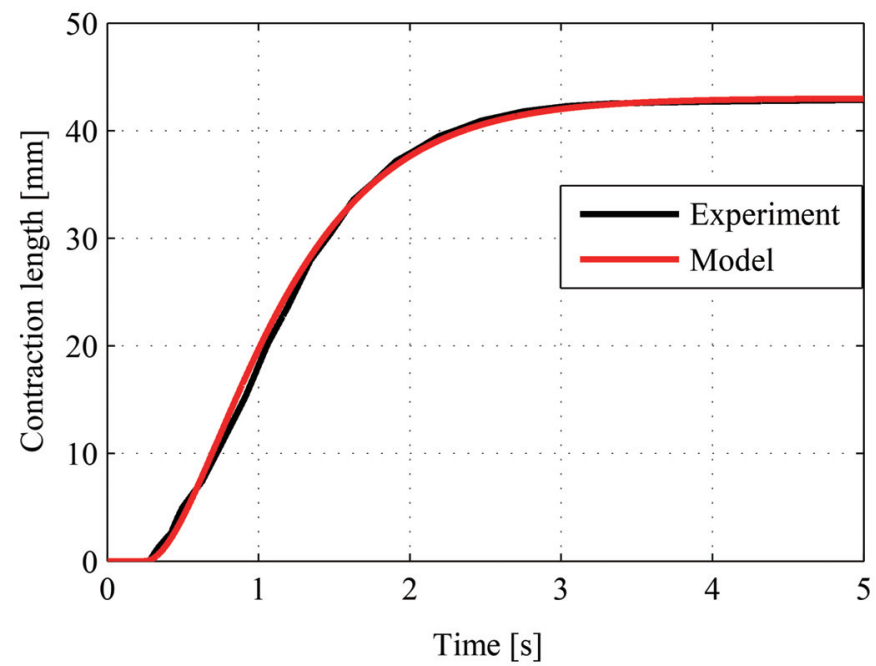

Fig. 7. Response of pressurizing.

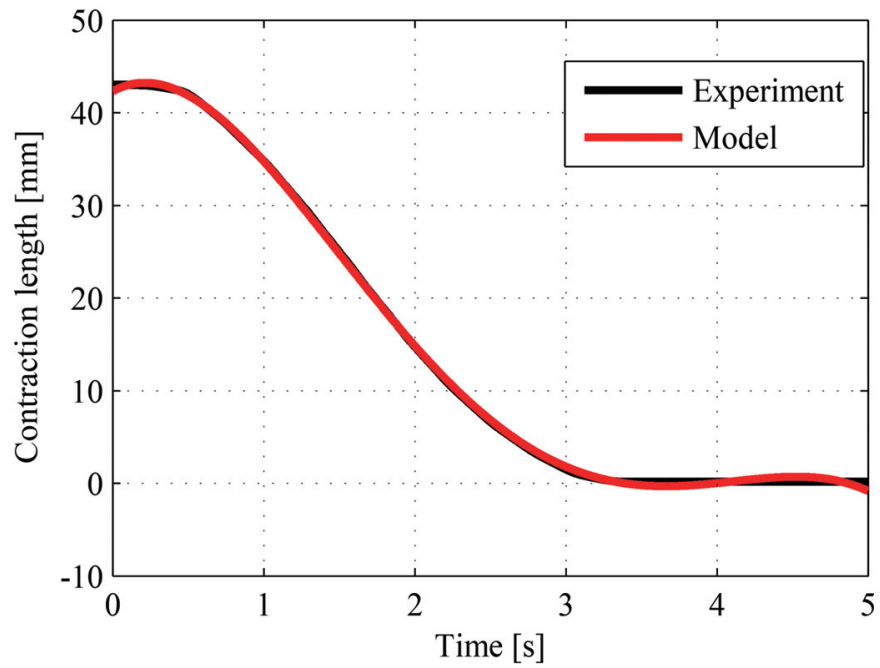

Fig. 8. Response of depressurizing.

Through further comparisons, we suppose the control model of pressurizing to be a second-order system with pure time delay element. And the transfer function is described by the following expression.

$$
G(s)=\frac{K}{\left(T_{1} s+1\right)\left(T_{2} s+1\right)} e^{-\tau s}
$$

In this model, there are totally four parameters that need to be identified: the proportional factor $K$, the time constants $T_{1}, T_{2}$ and $\tau$. Assume a set of values for these parameters, and figure out the step response curve using Matlab. Then, according to the difference of the experimental and model curves, adjust the 


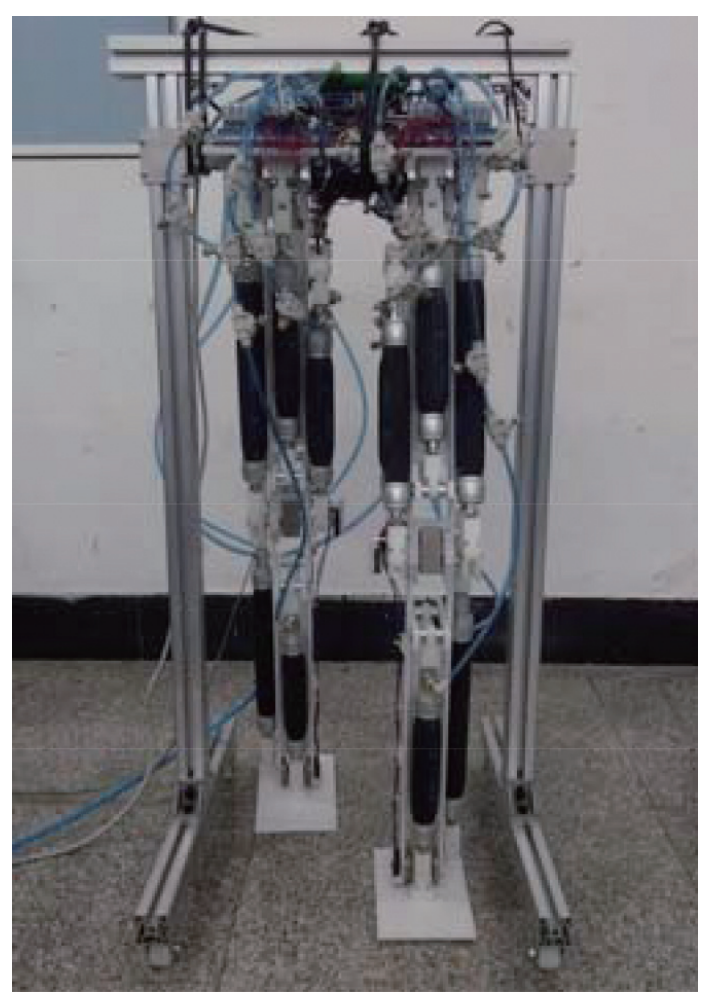

Fig. 9. Experimental setup.

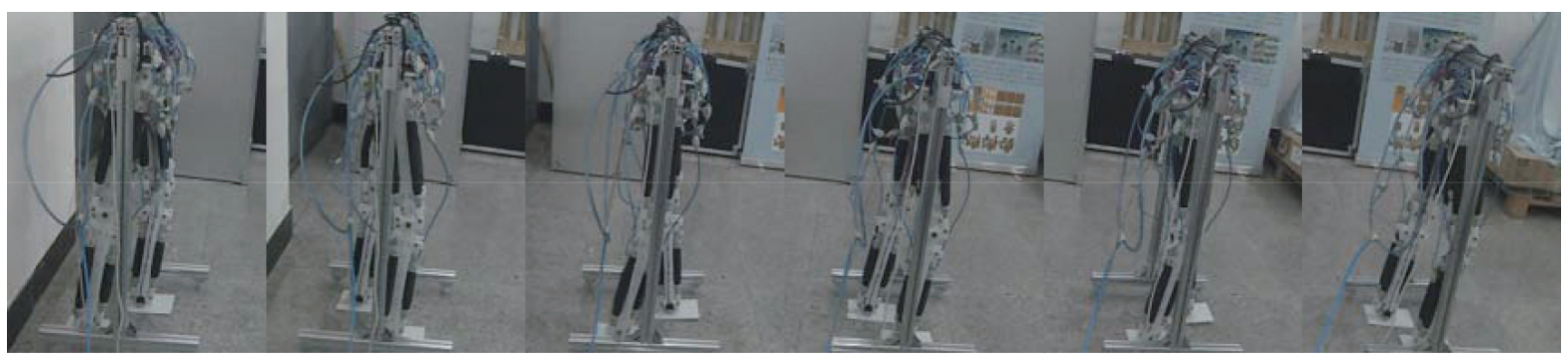

Fig. 10. Screenshots of walking.

parameters constantly until the satisfying result is reached, presented as follows.

$$
G(s)=\frac{L(s)}{P(s)}=\frac{43}{(0.49 s+1)(0.48 s+1)} e^{-0.25 s}
$$

The step response of the identified model is plotted as the red curve in Fig. 7, indicating that the model has high fitting degree.

After the model is obtained, a PID controller is designed to achieve fast and accurate response for the second-order system. To determine the proportional gain $K_{P}$, integral gain $K_{I}$, and differential gain $K_{D}$ of the PID controller, a simulation is conducted. As the result, the appropriate values of $K_{P}, K_{I}$ and $K_{D}$ are $0.05,0.05$ and 0.013 respectively. Thus the agonistic muscle can be controlled to contract using the PID controller. 
For the depressurizing process, the measured contraction length of the muscle versus time is plotted as the black curve in Fig. 8. Unlike the pressurizing process, polynomial fitting technique is applied to fit the curve to derive the relationship between the contraction length and time. After several trials, the polynomial with the highest fitting degree is obtained and presented as:

$$
\Delta L=-0.6157 t^{4}+6.895 t^{3}-22.55 t^{2}+8.707 t+42.33
$$

The curve of the identified model is plotted as the red curve in Fig. 8, which has high fitting degree. Then on the basis, the antagonistic muscle can be controlled to achieve the desired relaxation by adjusting the open and close time of the solenoid valve.

\section{Walking experiments}

Having designed the biped robot and its controller, walking experiments are carried out to test the validity. The biped robot has only six DOF around the pitch axis, which means that its movements are constrained in the sagittal plane in theory. However, actually because of the changing position of the gravity center, the real biped robot will swing in the lateral plane when it moves forward. Obviously, the coupled motion will directly influence the walking stability of the biped robot. Thus, the robot is placed in a cart that confines it to the sagittal plane, as shown in Fig. 9. Bungee cords prevent the robot from falling, but do not provide any forward propulsive force to the step.

Following observation in biomechanics, we assume that monoarticular muscles contribute to generate the power needed by the movements, whereas biarticular muscles mainly contribute to transfer force between joints. Therefore, the monoarticular muscles are controlled by the walking control system to follow the pre-determined joint motion, while the biarticular muscles are not actively controlled during walking. The valves for biarticular muscles are kept closed during experiments after initial supply. Snapshots of the biped robot walking cycle are shown in Fig. 10. The step length is $500 \mathrm{~mm}$, and the walking cycle is $8 \mathrm{~s}$. The results show that the robot is able to walk with the proposed control strategy.

\section{Discussion and conclusions}

In this paper, a bipedal walking robot actuated by PAMs is proposed. Its mechanical structure is designed by mimicking the musculoskeletal architecture of human lower limbs. High-speed on/off solenoid valves are adopted to control the muscles in order to reduce the system cost and weight. The control models of pressurizing and depressurizing the muscles are derived respectively through parameter identification experiments. The control scheme is designed based on the models and then verified by walking experiments.

However, the current realized walking is rather slow. One of the possible reasons is the limitation of gas flow, because the compressed air is supplied to all the valves through $8 \mathrm{~mm}$ diameter tube from a single external compressor. Meanwhile, the compressor limits the motion of the biped robot. If we want to get the robot fully self-contained, it tends to have a high requirement against the pressure and flow of the air source. What's more, the hysteresis of the muscles is tested to be as large as $0.25 \mathrm{~s}$, making it difficult for the robot to realize fast dynamic movements such as running, continuous jumping and hopping. And real-time online stability control under disturbances also requires a quick response of actuators. On the other hand, the biped robot has a degree of compliance owing to the muscles. The foot of the robot is desired to be flat when it makes contact with the ground. In the experiment, we found that 
even though the foot was not parallel to the floor at initial contact, the ankle joint could still help keep it flat.

In the future, we plan to replace the PAMs with servo motors for the hip joints of the biped robot. Because fast and accurate hip joint motion plays an important role in achieving stable dynamic tasks. For the knee and ankle joints, we think they do not necessarily require accurate positioning but rather compliance. So PAMs will still be considered as actuators. In this way, the advantages of electrics and pneumatics can be combined, making it hopeful to develop a versatile and compliant musculoskeletal biped robot which supports a wide range of motion modes. In addition, more advanced control techniques will also be studied and implemented on the robot.

\section{Acknowledgements}

The work reported in this paper is supported by National Magnetic Confinement Fusion Science Program "Multi-Purpose Remote Handling System with Large-Scale Heavy Load Arm" (grant 2012GB102004) and the project of the State Key Laboratory of Robotics and System (grant SKLRS201304B).

\section{References}

[1] P. Anton, S. Juraj. The use of pneumatic artificial muscles in robot construction. Industrial Robot: An International Journal. 2011; 38(1): 11-19.

[2] G. Belforte, G. Eula, S. Appendino. Design and development of innovative textile pneumatic muscles. The Journal of The Textile Institute. 2012; 103(7): 733-743.

[3] D. Villegas, M.V. Damme, B. Vanderborght, P. Beyl, D. Lefeber. Third - Generation pleated pneumatic artificial muscles for robotic applications: development and comparison with McKibben muscle. Advanced Robotics. 2012; 26: 12051227.

[4] Y. Nakanishi, T. Izawa, M. Osada, N. Ito, S. Ohta, J. Urata, M. Inab. Development of musculoskeletal humanoid Kenzoh with mechanical compliance changeable tendons by nonlinear spring unit. In: Proceedings of the 2011 IEEE International Conference on Robotics and Biomimetics, Phuket, Thailand. 2011, pp. 2384-2389.

[5] Y. Nakanishi, Y. Asano, T. Kozuki, H. Mizoguchi, Y. Motegi, M. Osada, T. Shirai, J. Urata, K. Okada, M. Inaba. Design concept of detail musculoskeletal humanoid "Kenshiro" - toward a real human body musculoskeletal simulator. In: Proceedings of the 2012 12th IEEE-RAS International Conference on Humanoid Robots, Osaka, Japan. 2012; pp. 1-6.

[6] B. Vanderborght, R. Ham Van, B. Verrelst, M. Damme Van, D. Lefeber. Overview of the Lucy project: dynamic stabilization of a biped powered by pneumatic artificial muscles. Advanced Robotics. 2008; 22(10): 1027-1051.

[7] B. Verrelst, J. Vermeulen, B. Vanderborght, R. Ham Van, J. Naudet, D. Lefeber, F. Daerden, M. Damme Van. Motion generation and control for the pneumatic biped "Lucy". International Journal of Humanoid Robotics. 2006; 3(1): 67-103.

[8] B. Vanderborght, B. Verrelst, R. Ham Van, M. Damme Van, R. Versluys, D. Lefeber. Treadmill walking of the pneumatic biped Lucy: walking at different speeds and step-lengths. International Applied Mechanics. 2008; 44(7): 134-142.

[9] K. Hosoda, K. Narioka. Synergistic 3D limit cycle walking of an anthropomorphic biped robot. In: Proceedings of the 2007 IEEE/RSJ International Conference on Intelligent Robots and Systems, San Diego, CA, USA. 2007; pp. 470-475.

[10] K. Hosoda, T. Takuma, A. Nakamoto, S. Hayashi. Biped robot design powered by antagonistic pneumatic actuators for multi-modal locomotion. Robotics and Autonomous Systems. 2008; 56: 46-53.

[11] K. Narioka, K. Hosoda. Designing synergistic walking of a whole-body humanoid driven by pneumatic artificial muscles: an empirical study. Advanced Robotics. 2008; 22: 1107-1123.

[12] K. Narioka, S. Tsugawa, K. Hosoda. 3D limit cycle walking of musculoskeletal humanoid robot with flat feet. In: Proceedings of the 2009 IEEE/RSJ International Conference on Intelligent Robots and Systems, St. Louis, USA. 2009; pp. 4676-4681.

[13] K. Ogawa, K. Narioka, K. Hosoda. Development of whole-body humanoid "Pneumat-BS" with pneumatic musculoskeletal system. In: Proceedings of the 2011 IEEE/RSJ International Conference on Intelligent Robots and Systems, San Francisco, CA, USA. 2011; pp. 4838-4843.

[14] K. Narioka, T. Homma, K. Hosoda. Humanlike ankle-foot complex for a biped robot. In: Proceedings of the 201212 th IEEE-RAS International Conference on Humanoid Robots, Osaka, Japan. 2012; pp. 15-20. 
[15] K. Narioka, T. Homma, K. Hosoda. Roll-over shapes of musculoskeletal biped walker. At-Automatisierungstechnik. 2013; 61(1): 4-15.

[16] R. Niiyama, Y. Kuniyoshi. Design principle based on maximum output force profile for a musculoskeletal robot. Industrial Robot: An International Journal. 2010; 37(3): 250-255.

[17] M. Shimizu, K. Suzuki, K. Narioka, K. Hosoda. Roll motion control by stretch reflex in a continuously jumping musculoskeletal biped robot. In: Proceedings of the 2012 IEEE/RSJ International Conference on Intelligent Robots and Systems, Algarve, Portugal. 2012; pp. 1264-1269.

[18] X. Liu, A. Rosendo, M. Shimizu, K. Hosoda. Improving hopping stability of a biped by muscular stretch reflex. In: Proceedings of 2014 14th IEEE-RAS International Conference on Humanoid Robots, Madrid, Spain. 2014; pp. 658-663.

[19] A. Rosendo, X. Liu, M. Shimizu, K. Hosoda. Stretch reflex improves rolling stability during hopping of a decerebrate biped system. Bioinspiration \& biomimetics. 2015; 10(1): 016008.

[20] H. Iijima, K. Sayama, H. Masuta, A. Takanishi, H. Lim. Mechanism of one-legged jumping robot with artificial musculoskeletal system. In: Proceedings of 2013 13th International Conference on Control, Automation and Systems, Gwangju, Korea. 2013; pp. 869-874.

[21] B. Tondu. Modeling of the McKibben artificial muscle: A review. Journal of Intelligent Material Systems and Structures. 2012; 23(3): 225-253

[22] G. Wang, N.M. Wereley, T. Pillsbury. Non-linear quasi-static model of pneumatic artificial muscle actuators. Journal of Intelligent Material Systems and Structures. 2015; 26(5): 541-553.

[23] F. Sorge. Dynamical behavior of pneumatic artificial muscles. Meccanica. 2015; 50: 1371-1386.

[24] M.A. Lewis, T.J. Klein. A robotic biarticulate leg model. In: Proceedings of the 2008 IEEE Biomedical Circuits and Systems Conference, Baltimore, MD, USA. 2008; pp. 57-60.

[25] T.J. Klein, M.A. Lewis. A robot leg based on mammalian muscle architecture. In: Proceedings of the 2009 IEEE International Conference on Robotics and Biomimetics, Guilin, China. 2009; pp. 2521-2526. 